\title{
Computer Simulation Technology Application in Hydraulic System Analysis and Design
}

\author{
Hu Xiaoping* \\ School of Mechanical Engineering \\ Lishui University \\ Lishui,323000,China \\ e-mail: zjlsxp@126.com
}

\author{
You Zhangping \\ School of Mechanical Engineering \\ Lishui University \\ Lishui,323000,China \\ e-mail:youzping@163.com
}

\begin{abstract}
With the development of mechanics and computer technology, computer simulation has become an important tool in the hydraulic system analysis and design. The application of computer simulation technology in hydraulic system is introduced in this paper. A hydraulic servo system was modeled by standard procedures. By simple operation of the software, named MATLAB/Simulink, dynamic characteristics of the hydraulic servo system are simulated. The result of the simulation verifies the validity of the method, which is applied in research.
\end{abstract}

Keywords- computer simulation technology; application; hydraulic system; dynamic characteristics

\section{INTRODUCTION}

The field of Computer Simulation has enjoyed rapid advances in recent years. computer technology and simulation technology is the combination of computer simulation technology as a new method of scientific research applied to various fields, used to solve the problems of pure mathematical methods or practical experiments cannot be solved, has a positive role in promoting the formation of scientific research and technological achievements.

Hydraulic simulation technology has been the interesting research topic for about 30 years. The wide variety of applications of complex hydraulic system requires easy modeling and simulation techniques. Up to now, the dynamic modeling and analysis of fluid pipeline networks have been studied with the method of difference equation ${ }^{[1]}$, ${ }^{[2]}$, differential equation ${ }^{[3], ~[4]}$ transfer function ${ }^{[5], ~[6]}$. These methods are applied widely in all sorts of advanced control strategies ${ }^{[7] \sim[9]}$ to analysis of stability and frequency response of hydraulic system, but are inefficient in dealing with the nonlinear problem which is ubiquity in complex hydraulic system. In order to analysis the influence of the structure parameters on system dynamic characteristics efficiently, MATLAB/Simulink software is used in complex hydraulic system. Based on the software, an efficient approach for modeling and simulation of dynamic characteristics of complex hydraulic system is proposed. To illustrate this method, an example hydraulic servo system is modeled and simulated.

\section{Establishments Mathematical Model}

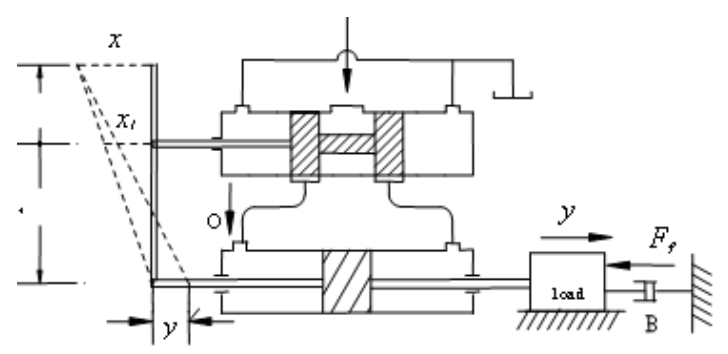

Figure 1. a hydraulic servo system

Take the four-way valve in Fig. 1 as study objects, the equation of flow characteristic is given:

(1) Continuity equation of hydraulic cylinder can be obtained from continuity equation of compressible fluid:

$$
\mathrm{Q}=\mathrm{k}_{\mathrm{q}} \mathrm{x}_{1}-\mathrm{k}_{\mathrm{L}} \mathrm{P}_{\mathrm{L}}
$$

Where $\mathrm{Q}$ is load flow, $\mathrm{m}^{3} \cdot \mathrm{s}^{-1} ; k_{\mathrm{q}}$ is the flow gain of slide valve near the operating point of stable state, $m^{2} \cdot s^{-1} ; x_{1}$ is the spool displacement, $m ; p_{\mathrm{L}}$ is the load pressure drop, $\mathrm{Pa} ; k_{\mathrm{L}}$ is the flow-pressure coefficient of slide valve near the operating point of stable state, $m^{3} \cdot(\mathrm{Pa} \cdot \mathrm{s})^{-1}$.

$$
Q=A \frac{d y}{d t}+\frac{V_{0}}{2 \beta} \cdot \frac{d p_{L}}{d t}+k_{m} p_{L}
$$

Where $A$ is the effective area of piston, $m^{2} ; y$ is piston displacement , $m ; k_{m}=l_{m}+\frac{1}{2}\left(k_{l}+l_{0}\right)$ is the total coefficient of leakage, among which, $l_{\mathrm{m}}$ is the leakage coefficient of hydraulic cylinder, $l_{0}$ is the external leakage coefficient of valve-controlled cylinder system, $m^{3} \cdot(\mathrm{Pa} \cdot \mathrm{s})^{-1} ; V_{0}$ is the total volume of tow oil chambers, $m^{3}$; $\beta$ is the effective fluid volumetric modulus of elasticity, $\mathrm{N} \cdot \mathrm{m}^{-2}$.

(2) If the weight of oil and nonlinear loads such as coulomb friction are neglected, according to Newton's second law, equilibrium equation of the hydraulic system can be expressed as

$$
A p_{L}=m \cdot \frac{d^{2} y}{d t^{2}}+B \frac{d y}{d t}+F_{f}
$$

Where $m$ is the total mass of piston and load, $\mathrm{kg}$; $B$ is the viscous damping coefficient of piston and load, N.S.m ${ }^{-1} ; F_{\mathrm{f}}$ is the load force which acts on piston. 
According to the geometrical relationship of stick position in Fig. 1, equation of opening amount of the valve is given on motion:

$$
x_{1}=\frac{b}{a+b} x-\frac{a}{a+b} y
$$

Where $a$ or $b$ is the two-stage length of joystickdivided by fulcrum(shown in Fig.1),m.From Eqns. (1) and (4), it can be derived by Laplace transforms and linearization that

$$
\begin{gathered}
P_{L}=\frac{\frac{K_{q} b}{a+b} X-\left(A S+\frac{K_{q} a}{a+b}\right) Y}{\frac{V_{0}}{2 \beta} S+K_{L}+K_{m}} \\
Y=\frac{A P_{L}-F_{f}}{S(m S+B)}
\end{gathered}
$$

\section{Simulation}

From the mathematical models which are shown in Eqns. (5) and (6), the block diagrams of the hydraulic servo system can be drawn in Fig. 2.

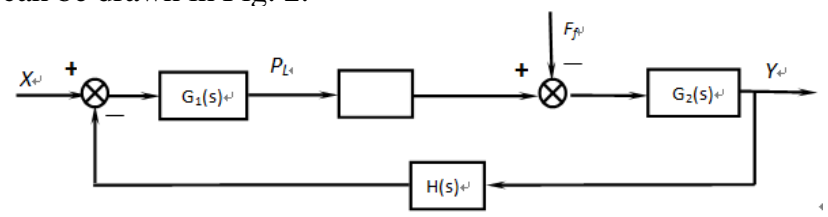

Figure 2. block diagram of the hydraulic servo system

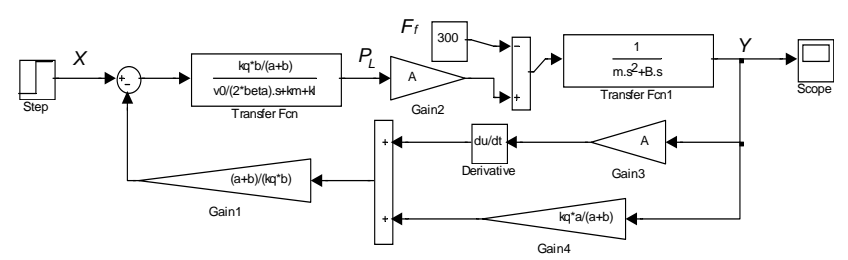

Figure 3. Simulation Model

Simulation model can be obtained from Fig. 2, as is shown in Fig. 3.Based on this; the equation solver named ode23 is applied to simulation calculation. Then by simple operation of SIMULINK, dynamic response of the hydraulic servo system is simulated in this paper. The parameters used for the simulation are shown in Table 1.

TABLE I. SIMULATION PARAMETERS

\begin{tabular}{|c|c|c|c|c|c|c|}
\hline $\boldsymbol{a}$ & $\boldsymbol{b}$ & $\boldsymbol{K}_{\boldsymbol{q}}$ & $\boldsymbol{V}_{\boldsymbol{0}}$ & $\boldsymbol{\xi}_{\boldsymbol{h}}$ & $\boldsymbol{K}_{\boldsymbol{L}}$ & $\boldsymbol{\beta}$ \\
\hline 0.5 & 0.5 & 0.124 & 0.00273 & 1.05 & $2.04 \times 10^{-11}$ & $1.4 \times 10^{9}$ \\
\hline$B$ & $m$ & $A$ & $F_{f}$ & $\omega_{h}$ & $K_{m}$ & \\
\hline 40000 & 2000 & 0.00268 & 300 & 26.9 & $1.495 \times 10^{-11}$ & \\
\hline
\end{tabular}

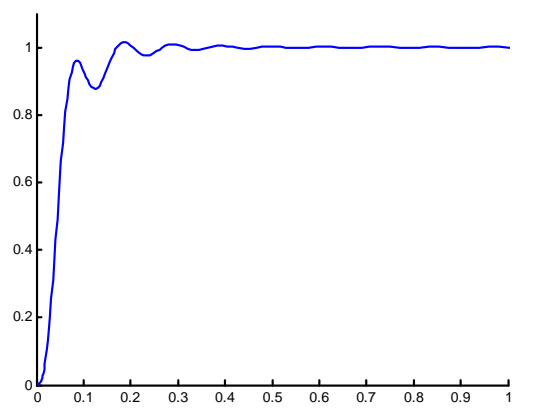

Figure 4. simulation result

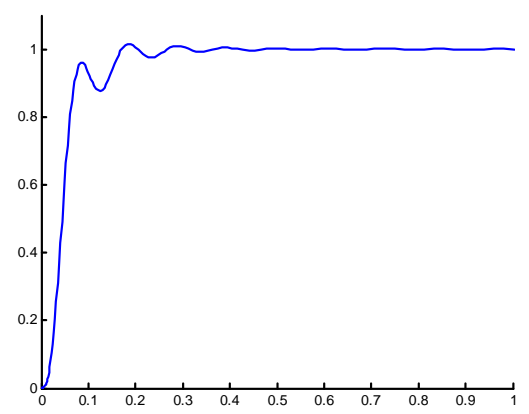

a) When $V_{0}$ decreased to $0.00191 \mathrm{~m}^{3}$

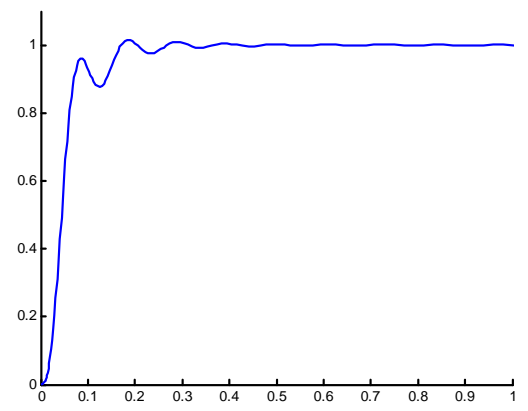

b) When $\mathrm{K}_{\mathrm{q}}$ decreased to $0.1054 \mathrm{~m}^{2} \cdot \mathrm{s}^{-1}$

Figure 5. the influence of parameters changed

The result is shown in Fig. 4. From the curve (Fig. 4), we can see obviously that there is initially an oscillation under the step input. After 0.45 seconds, response of the system is gradually stabilized. The new simulation results are shown in Fig. 5 with parameters changed. By comparing the results of Fig. 4 and Fig. 5, the influence of parameters changed on dynamic characteristics of system is investigated:

1) When $V_{0}$ which is cumulative volume of two oil cavities decreases, the systematic response time is significantly reduced; it is 0.32 seconds in Fig. 5a.Therefore, decreasing $V_{0}$ can improve the stability of the system.

2) When $K_{q}$ decreases, the systematic response time is significantly reduced too; it is 0.36 seconds in Fig. 5a.The result shows that dynamic characteristics of the hydraulic 
servo system under low flow gain is better than those under high flow gain.

\section{Conclusions}

1) From the Dynamic simulation process, if the method proposed with Simulink is applied in dynamic characteristics of complex system; complex program need not be compiled. This is reliable and simple because of direct simulation with mathematic model.

2) From the Dynamic simulation result, the method proposed with Simulink is an accurate and practical approach for dynamic simulation of complex hydraulic system.

\section{ACKNOWLEDGMENT}

We appreciate the support from Zhejiang Provincial Natural Science Foundation of China under Grant No. LZ12F02001; We also appreciate the support from Zhejiang Province's Key Disciplines of Mechanical Design and Theory, Zhejiang Sci.-Tech. University Key Lab ( ZSTUMD 2011A005).

\section{REFERENCES}

[1] Kalyan R. A stochastic differential equation analysis of cerebrospinal fluid dynamics [J]. Fluids and barriers of the CNS,89, (2011),pp. 8-9

[2] Yang, G; GAO, J; Chen, B. Computer simulation of controlled hydraulic impactor system [J]. Advanced Materials Research, 180,(2011), pp. 122-127,.

[3] Hiroyuki H, Miiko I, Nobutoshi S.Solution functions of PDEQSOL (Partial differential EQuation SOlver language) for fluid problems , Proceedings of the 1991 ACM/IEEE conference on Supercomputing, 1991, pp.218-227

[4] Yuki S. Analysis of Two Partial Differential Equation Models in Fluid[D].Cambridge University Press,2009.

[5] Amirante,G. Del Vescovo,A. Lippolis. Evaluation of the flow forces on an open centre directional control valve by means of a computational fluid dynamic analysis .Energy Conversion and Management, 8, (2006), ,pp:1748-1760 .

[6] Ditya A,Gyula S,Gabor K. Translation of Simulink/Stateflow Models to Hybrid Automata Using Graph Transformations [J].Electronic Notes in Theoretical Computer Science, 109 , (2004), pp.43-56 .

[7] Wang C H, Tsai C H, Lin W S.Robust fuzzy model-following control of robot manipulators[J].IEEE Trans. Fuzzy Syst, 8 (2000),pp.462469.

[8] Zhang Xiaodong, Jia Qingxuan, Sun Hanxu , Chu Ming. The Research of Space Robot Flexible Joint Trajectory Control[J]. Journal of Astronautics in Chinese. 29 (2008), pp. 1865-1869.

[9] Lewis F L, Kim Y H. Intelligent optimal control of robotic manipulators using neural networks [J].Automatica, 36 (2000),pp.1355-1364. 